\title{
Guardianas de la sabiduría: Pedagogía de los cantos ancestrales del pueblo Embera Eyábida
}

\author{
$\longrightarrow$ \\ Wisdom Keepers: Pedagogy of Ancestral Songs from the \\ Indigenous People Embera Eyábida \\ Nataly Domicó-Murillo* \\ (D): https://orcid.org/0000-0002-9644-6503
}

Tipo de Artículo: Informes de Investigación y ensayos inéditos

Doi: 10.17533/udea.unipluri.19.1.04

Cómo citar este artículo:

Domicó-Murillo, N. (2019). Guardianas de la sabiduría: Pedagogía de los cantos ancestrales del pueblo Embera Eyábida. Uni-pluriversidad, 19(1), 56-71, Doi: https://doi.org/10.17533/udea.unipluri.19.1.04

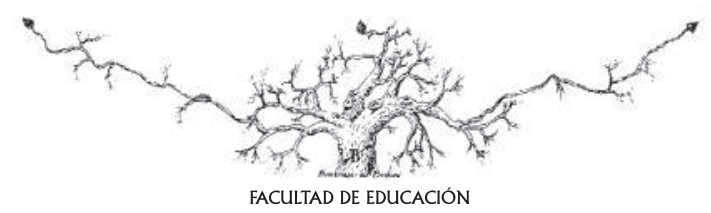

Recibido: 2019-05-29 • Aprobado: 2019-06-10

* Asesora Pedagógica del Cabildo Mayor Indígena de Chigorodó. Licenciada en Pedagogía Infantil. Integrante del grupo de Investigación Diverser de la Facultad de Educación de la Universidad de Antioquia.

E-mail: nataly.domicom@udea.edu.co. 


\title{
Resumen
}

Este artículo presenta los resultados de la investigación desarrollada desde y con el pueblo Embera Eyábida de Chigorodó sobre los cantos ancestrales de las mujeres y su vinculación con los espacios pedagógicos y didácticos de las escuelas indígenas. Como pregunta de investigación se planteó ¿Qué elementos pedagógicos pueden aportar los cantos ancestrales al modelo de Educación Propia que se ejerce dentro de las escuelas indígenas de la comunidad Embera Eyábida? El objetivo de la investigación fue recuperar cantos ancestrales o truambis de las mujeres abuelas de este pueblo indígena, para construir material pedagógico que aporte a la Educación Propia de los niños y niñas. El trabajo se inscribió dentro del paradigma de investigación indígena sustentado por Linda Tuhiwai Smith y profundizó en el método de investigación Embera adelantado por Baltasar Mecha, lingüista indígena del pueblo Embera Dobida. Se hallaron cinco tipos de cantos ancestrales o truambis en el pueblo Embera de Chigorodó: de animales, sanación, duelo, arrullos y parejas. Estos cantos se encuentran en riesgo de desaparecer si no se realizan procesos pedagógicos que fortalezcan y visibilicen su práctica. Esto se debe a la amenaza del sistema dominante que desvirtúa los intereses de la niñez y de la juventud Embera, enrutándolos a asimilar perspectivas occidentales en sus prácticas culturales que afectan el arraigo identitario. En la actualidad, el grupo poblacional que practica y enseña estos conocimientos está integrado por las mujeres Embera Eyábida, quienes a su vez son guardianas y pedagogas de la sabiduría ancestral. Se hace urgente reconocer y profundizar en sus saberes para fortalecer la memoria comunitaria del pueblo Embera.

Palabras clave: Educación Propia, Comunidad, Sabedoras, Cultura, Investigación Indígena.

\begin{abstract}
This article presents the results of a research developed by and with the indigenous people Embera Eyábida from Chigorodó. It deals with ancestral songs by the women and their connection with pedagogical and didactic spaces of the indigenous schools. The research question is: what pedagogical elements can ancestral songs contribute to the model of Self-Education promoted in indigenous schools of the Embera Eyábida? The objective of research was to recover ancestral songs or truambis sung by grandmothers of the indigenous people and to build pedagogical material that contributes to children's education. This work was based on the paradigm of indigenous research by Linda Smith and also on the Embera research methodology by Baltasar Mecha, an Embera Dobida indigenous linguist. Five types of ancestral songs or truambis were found in the Embera Village of Chigorodó: related to animals, healing, grief, lullabies, and couples. These ancestral songs are in danger of
\end{abstract}


disappearing if pedagogical processes are not carried out in order to strengthen and make visible their practice. This is due to the dominant system threatens to distort the interests of Embera childhood and youth and influence them to assimilate western perspectives that affect their cultural identity. Currently the population group that practices and teaches this knowledge is primarily the Embera Eyábida women. They are both keepers and pedagogues of ancestral wisdom. It is urgent to recognize and delve into their knowledge in order to strengthen the collective memory of the Embera indigenous people.

Keywords: Autonomous Education, Community, Indigenous Elders, Culture, Indigenous Research 


\section{INTRODUCCIÓN}

Los pueblos originarios de Colombia se encuentran en nuevas reconquistas. No han cesado las acciones de despojo, exterminio $\mathrm{y}$ aculturación que trajeron los españoles con su llegada. El proyecto capitalista-moderno en el que varios países del mundo, entre ellos Colombia, sustentan hoy su proyecto político y económico ejerce, al igual que en la época colonial, acciones de violación a los derechos humanos, desigualdades, etnocidio de baja intensidad y exclusión, en especial a las personas y comunidades que aún viven en la ruralidad.

El pueblo Embera Eyábida se encuentra en condiciones de fragilidad frente a las hegemonías del sistema capitalista, por ser un pueblo que históricamente ha habitado territorios selváticos y montañosos ricos en biodiversidad y recursos naturales. En consecuencia, las comunidades Embera han sido objeto de múltiples desplazamientos forzados, aculturación sistemática y violencias de naturaleza militar y paramilitar dentro de sus territorios, realidad documentada por el Observatorio del Programa Presidencial de Derechos Humanos y DIH (2010):

Frente al panorama de los grupos armados existentes en la zona, así como la riqueza hidrográfica en la región del Nudo de Paramillo, la violencia se ha presentado como uno de los principales problemas para la comunidad Embera Katío \{Embera Eyábida\}. En este contexto, las acciones de estas agrupaciones han causado asesinatos de líderes, desapariciones, quemas de viviendas, desplazamientos y reclutamientos a lo largo de estos años (Diagnóstico de la situación del pueblo indígena Embera Katío. (p. 15)
Pese a los acuerdos internacionales como el Convenio 169 de la OIT, la Declaración de las Naciones Unidas sobre los Derechos de los Pueblos Indígenas y, a nivel nacional, la Constituyente de 1991 y el más reciente Decreto Autonómico 1953 de 2014, las condiciones de marginalidad y desventaja económica, cultural y epistémica continúan latentes, afectando de forma profunda el bienestar del pueblo Embera Eyábida.

En Antioquia existen 4 pueblos indígenas reconocidos por el Ministerio del Interior: Embera Chamí, Embera Eyábida, Gunadule y Zenúes, pueblos portadores de legados ancestrales y una gama de prácticas culturales e identitarias que se contraponen a las propuestas hegemónicas que el capitalismo actual defiende.

Este proyecto de investigación se desarrolló con el pueblo Embera Eyábida, en jurisdicción del Cabildo Mayor Indígena de Chigorodó (En adelante, CMICH). Actualmente, en las comunidades indígenas de este municipio se presentan problemas en torno a la educación escolarizada, ya que las estructuras curriculares de los planes de estudios se basan en conocimientos occidentales y externos a la cultura propia.

Como resultado, parte de las nuevas generaciones no se encuentran interesadas en aprender las prácticas culturales originarias del pueblo Embera, incrementando el uso preferencial de la lengua castellana y haciendo más notoria la mezcla de estos códigos con su lengua nativa. Además, parte de los comuneros están migrando de las comunidades por presiones exógenas o por libre 
elección hacia los centros urbanos o pueblos alejados de sus resguardos.

Sin embargo, el pueblo Embera Eyábida aún conserva prácticas culturales ancestrales. En este caso, los cantos o Truambis son una práctica en peligro de desaparecer. Por lo tanto, vincularlos a los espacios pedagógicos de las escuelas fue una acción que se determinó como urgente, siendo vital realizar un análisis e interpretación de estos cantos, tarea que enmarcó el propósito central de este trabajo de investigación.

\section{Pazake Ka Kirinchata Emberara: Trenzando el PENSAMIENTo de las abuelitas Embera Eyábida. AnÁlisis de los Truambis o cantos ANCESTRALES}

La música de muchos pueblos indígenas de Colombia no cabe en estructuras lineales convencionales que se condicionan en tiempos y ritmos universalizados. La música embera se crea en la relación íntima que hay entre el hombre, la mujer, la naturaleza y los sonidos que cada uno alberga. Es decir, existe un vínculo estrecho entre la melodía y el ser Embera. La música y el canto recrean varias actividades culturales como los rituales, festividades y cotidianidades familiares que son expresadas a través de esta manifestación cultural, a ella se articulan también los movimientos y sonidos de los seres que habitan en la selva. "La música de los grupos indígenas está íntimamente ligada a la vida tradicional y espiritual, y mientras se mantiene la religión tradicional la música asociada a ella pervive" (Bermúdez, 1987, p. 86).

La música en el pueblo Embera tiene dos vertientes, por un lado, se encuentra la música instrumental que es utilizada para las danzas y las festividades y tiene como base instrumentos autóctonos. "Desde la flauta surge nuestra música tradicional, se interpreta y acompaña con diferentes instrumentos como el tambor y las maracas" (CMICH, 2013, p.40).
Por otro lado, existe la música ancestral que se manifiesta a través de la voz y se llama naturalmente truambi o canto ancestral. Esta práctica se realiza en los rituales de sanación dirigidos por un Jaibana ${ }^{1}$ o una Jaibanawera $^{2}$ y por parte de las mujeres Embera en diferentes escenarios de la vida en comunidad. En el libro Lo que los ancestros nos dejaron: nuestra identidad Embera en Chigorodó se expone que:

Un canto muy importante y tradicional para nosotros es el Truambi, la forma tradicional que tenemos para expresar nuestros pensamientos, sentimientos y emociones. Son narraciones melodiosas, cortas, lentas, enseñadas por las mujeres adultas, quienes tienen el rol de transmitirlo de generación en generación, su ritmo es un punto entre poesía recitada y un canto. (CMICH, 2013, p. 40)

Los truambis son melodías vocales utilizadas dentro de la cultura para narrar contenidos relacionados con la vida y la existencia Embera. En ellos se incluye la relación con la naturaleza, los sentimientos y el pensamiento de las mujeres, los conflictos internos, onomatopeyas, especialmente de animales, etc. La cultura se gesta y dinamiza en estos contenidos. 


\section{¿Quiénes cantan los truambis?}

Jorge Domicó, gobernador local de la comunidad de Chigorodocito afirma que el hombre Embera cantaba Truambis anteriormente. Sin embargo, en la actualidad, esta práctica solo es ejercida por las mujeres, en particular por las mujeres abuelas o adultas mayores. Al respecto, el señor Domicó comenta que: "Los Embera cuando tomaban licor en las fiestas se inspiraban para componer truambis. Yo tenía un tío que cuando asistía a las fiestas y tomaba licor creaba cantos ancestrales largos para contar lo que estaba pasando en la comunidad y en su familia (Domicó, J. Comunicación personal, septiembre 20 de 2017).

En la actualidad, el canto ancestral masculino es practicado sólo por los Jaibanas que realizan truambis en sus ceremonias, es decir, en gran medida son las mujeres quienes mantienen viva esta práctica cultural. Sin embargo, no se puede afirmar que sea una constante histórica puesto que los hombres Embe$\mathrm{ra}^{3}$ han jugado un papel importante en la composición de algunos truambis que aún persisten. Diversas interpretaciones que escuché por parte de las mujeres entrevistadas fueron compuestas por hombres. Por otra parte, no se identificó a jóvenes, niñas o niños que supieran interpretar algún truambi; todas las conocedoras de esta práctica son adultas y, por eso se consideran a lo largo de esta investigación como las guardianas del canto ancestral.

\section{¿Cómo se aprende?}

\section{“¿Usted cómo aprendió? ¿Quién le en- señó?}

María Lina Bailarín: A través de los viejos, a través de mi madre.

\section{¿Usted preguntaba o escuchaba?}

M L B: Mi madre cantaba, cantaba para que aprendiéramos, así enseñaba a sus hijas. Después, mi mamá me enseñó a mí. Mi abuela enseñaba a mi mamá y mi mamá me enseñó a mí, a nosotras.

\section{¿Cómo le enseñaba, por la mañana o por la tarde?}

M L B: Por la tarde, cuando ya casi estábamos para acostarnos y en eso ella empezaba a cantar el truambi. No dormía por cantar. Así nos enseñaba para que más tarde enseñáramos a nuestros hijos y así lo decía; que truambi hay que enseñar a nuestros hijos o que hay que enseñar cantando; recordando de la vida que había pasado y cantar eso o cantar mencionando las cosas bonitas que tienen los ríos o de lo que hay en la tierra. Así nos decía ella o nos decía que cuando nos dejaban solas con nuestros hijos sufriendo por otra mujer que la ven bonita y se van, entonces que empezaban a decir todo eso en truambi.

\section{¿Usted aprendió así?}

M L B: Sí, así aprendí, así nos decía, así nos enseñó" (Bailarín, M, L. Comunicación personal, septiembre 9 de 2017).

La sabedora María Lina Bailarín explica de forma clara cómo se aprende el truambi: son las madres o abuelas las encargadas de compartir estos conocimientos con sus hijas. En consecuencia, se infiere que el silencio y la escucha, tal como lo resaltan los principios pedagógicos de la Licenciatura en Pedagogía de la Madre Tierra ${ }^{4}$, son habilidades vitales que deben desarrollarse para poder aprender sobre los cantos ancestrales. Otra habilidad que cumple un papel significativo y central en este aprendizaje es la oralidad: "Es importante reconocer y valorar el aporte 
del tejido de la tradición oral, ya que, por ella, hemos conocido la sabiduría de nuestros ancestros, que nos ha sido transmitido por nuestras abuelas y los abuelos" (Green, 2011, p. 209). Para los pueblos indígenas, la oralidad representa el corazón de compartir la palabra, es la acción por la cual pueden narrar la historia. El truambi es entonces esa melodía de la palabra, que otorga ritmo a los cuentos, tonada a las narraciones, a las leyendas y a la vida del Embera.

\section{Tipos DE CANTOS}

Los tipos de cantos son variados y expresan desde los sonidos de animales hasta las rupturas amorosas. Esta investigación permitió identificar los siguientes tipos de Truambis:

\section{Truambis de Animales.}

Los animales hacen parte de la vida cotidiana del pueblo Embera, por eso se convierten en tema de inspiración para las composiciones y enseñan a las mujeres a cantar, porque ellas imitan los sonidos de los animales en sus notas vocales. El sonido del bosque en conjunto con los animales también enseña a las mujeres Embera a perfeccionar sus tonalidades, permitiéndoles explorar las capacidades sonoras de sus voces.

La importancia de este tipo de cantos ancestrales radica en que son interpretaciones que las compositoras realizan de las acciones y características de los animales; algunas crean una mezcla entre los sonidos que emiten agregando a la composición características del animal. La autora del truambi "El pequeño loro" dijo al respecto:

"Esto no me lo enseñó nadie, sino que aprendi yo sola, un solo truambi. Sino que cuando el loro está furioso, sus ojos empiezan a encenderse como la luz del rayo. Entonces empecé a pensar para decir asi y asi aprendí. Eso ya hace dos años. Primero practiqué para ver si podia". (Domicó, G. Comunicación personal, septiembre 8 de 2017)

\section{Truambis de Sanación.}

En la cultura Embera, la sanación es comunitaria y colectiva. Para las ceremonias de curación de $J_{a i}{ }^{5}$ se realiza una especie de festejo al que son invitados varios amigos, familiares y vecinos para que acompañen al enfermo. No es solo un encuentro entre los seres físicos, es decir, entre los Emberas que asisten; el Jaibaná también invita a los espíritus de la naturaleza buenos o bonitos para que ayuden al paciente a sanarse. Así, los seres del mundo visible e invisible se unen para acompañar la sanación. El truambi juega un papel importante en este ritual porque es la forma en la que los espíritus escuchan el llamado del Jaibaná; a través del canto este les pide ayuda y acompañamiento.

Truambi de ritual de la Muerte o el duelo.

En muchas culturas, la muerte está mediada por acciones simbólicas que hacen de este proceso un ritual articulado a las manifestaciones y creencias comunitarias. El canto ancestral del pueblo Embera acompaña el ritual de la muerte, por ejemplo, en las velaciones se realizan cantos ancestrales para recordar la vida y la historia de la persona que partió.

\section{Truambi de Arrullo.}

Por lo general, el canto de arrullo es cantado por las madres, abuelas o tías para 
hacer dormir y calmar el llanto de los niños y niñas. La mujer embera tiene una fuerte articulación a la crianza de sus hijos, el vínculo desde su vientre gesta entre ella y el niño una conexión que perdura hasta la adolescencia. Las madres son las primeras pedagogas de los niños y niñas Embera Eyábida.

\section{Truambi de Pareja.}

Estos son los truambis que utiliza la mujer para enunciar situaciones de abandono y sufrimiento que viven en sus relaciones de pareja, a través de estos se lamentan y expresan sentimientos de tristeza. El abandono del hogar por parte de los hombres Embera aumenta cada día y es un impacto más del proceso de aculturación. A raíz de esta problemática- a la cual me he vinculado como activista- las mujeres Embera Eyábida de Chigorodó se están uniendo por medio del Comité de Mujeres ${ }^{6}$, exigiendo la participación política, formación académica y organizándose para crear acciones en conjunto con la jurisdicción especial indígena y aplicar sanciones o castigos que mitiguen los maltratos físicos, psicológicos y verbales ejercidos por los hombres Emberas dentro de la vida familiar y de pareja. Acciones que se articulan a los planteamientos del feminismo comunitario trabajado por mujeres indígenas de Guatemala y Bolivia, quienes exponen que:

“A proposta do feminismo comunitário compreende campos de ação e lutas como categorias para a ação política de fortalecimento das organizações de mulheres. Estes campos de ação e luta política permitem transformar as condições materiais da subordinação e exploração das mulheres em suas comunidades e sociedades" (Sacavino, 2017, p.115)
Este truambi expresa una caducidad de sentido con relación a las dinámicas actuales que enfrentan las mujeres Emberas. Hoy en día, si una mujer es abandonada por su marido, existe el mecanismo o espacio donde puede denunciar este hecho (Comité de Mujeres). Por otro lado, muchas de las mujeres abandonadas no optan por lamentarse y entregarse al sufrimiento, por el contrario, se organizan y asumen la responsabilidad de la economía de sus hogares; algunas trabajando la tierra, otras siendo maestras y participando en proyectos del Cabildo Mayor Indígena. Se puede afirmar que en estos tiempos la mujer Embera está posicionando su fuerza para mejorar su vida y la de su familia.

\section{Estado actual del truambi.}

Los modelos hegemónicos basados en intereses de los grandes capitales han ingresado de forma invisible pero fuerte en las comunidades indígenas. Este aspecto se puede evidenciar en diversos procesos como en el ingreso de modelos educativos conductistas, competitivos y progresistas dentro de las escuelas indígenas, y en las innovaciones tecnológicas utilizadas como consumo que sirven para la reproducción de discursos elitistas (televisión, celulares, tablets). Estos medios propagan y legitiman argumentos racistas, sexistas y excluyentes, posicionando un solo saber - poder con rezagos coloniales y una fuerte influencia neoliberal. Entonces en palabras de Quijano (2000) se trata de:

La colonialidad del poder, el capitalismo y el eurocentrismo. Por supuesto que este patrón de poder, ni otro alguno, puede implicar que la heterogeneidad histórico-estructural haya sido erradicada dentro de sus dominios. Lo que su globalidad implica es un piso básico de prácticas sociales comunes para todo el 
mundo, y una esfera intersubjetiva que existe y actúa como esfera central de orientación valórica del conjunto. Por lo cual las instituciones hegemónicas de cada ámbito de existencia social son universales a la población del mundo como modelos intersubjetivos. (p. 214215)

Los patrones productivos de las transnacionales y las multinacionales que tanto defiende la plutocracia colombiana y que históricamente han gobernado al país, ven a los territorios de las comunidades indígenas como lugares propicios para ejecutar sus proyectos extractivos de muerte. Por lo anterior, los pueblos somos afectados por múltiples pérdidas y deterioro de la tierra que deja como resultado dicho proyecto nocivo. La extracción no solo expropia la riqueza material, sino también la riqueza espiritual y cultural. Gran parte de la cosmogonía del pensamiento Embera se encuentra en completa conexión en espacio y tiempo con el territorio material, es decir, con la Madre Tierra.

Si no existiesen ríos limpios, animales selváticos, plantas medicinales, bosques, pensamiento y práctica Embera, los cantos ancestrales desaparecerían. La gran inspi- radora y pedagoga de las mujeres Embera cantadoras de truambi es la naturaleza y su relación directa con la cultura y la identidad. Si dicha naturaleza se extrae, maltrata y explota, afecta de forma categórica la relación que las mujeres han creado con ella y con los cantos ancestrales.

En definitiva, las condiciones en las que se encuentra actualmente los cantos ancestrales del pueblo Embera Eyábida de Chigorodó son de marginalidad y desventaja. Es desmotivador ver cómo se olvidan las abuelas conocedoras de esta práctica, los intereses de las juventudes son coartados por las ilusiones del modelo occidental y los niños y niñas están perdiendo los escenarios de aprendizajes comunitarios donde se fortalece la identidad cultural.

El canto ancestral se encuentra en un estado crítico frente a la continuidad de sus enseñanzas, las escuelas indígenas no poseen herramientas didácticas para propiciar su aprendizaje y los espacios educativos no formales que gestiona el $\mathrm{CMICH}$ se centran en temas políticos y jurídicos, restándole importancia a los temas culturales y por ende a los cantos ancestrales.

\section{AsPeCTOS PEDAgógicos Y DIDÁCTICOS}

Los análisis que se presentan en este apartado surgen de la entrevista realizada a la profesora Magnolia Tascón de los grados preescolar, primero, segundo y tercero de la escuela de la comunidad de Chigorodocito. A partir de la conversación y observación participante, se pudo inferir varios aspectos que determinan la cercanía o lejanía de la propuesta de Educación Propia de las escuelas indígenas de Chigorodó.
La profesora manifiesta que no existe una escuela como infraestructura, por ende, se dificulta realizar las clases de manera idónea. Los niños y niñas de la escuela de la comunidad de Chigorodocito reciben sus clases en la caseta comunitaria, espacio utilizado para las reuniones y asambleas, careciendo de dotación básica para la enseñanza (sillas, mesas, tableros, etc.). En el mismo espacio imparte las clases la maestra encargada de los grados cuarto y quinto. Investi- 
gadores e instituciones de la educación afirman que el ambiente escolar fortalece los procesos formativos y algunos autores sustentan que el ambiente escolar es un espacio simbólico, que trasciende lo físico.

Dentro del ámbito escolar, la mayoría de los docentes se centran en el aula como espacio protagonista de los aprendizajes, sin darse cuenta de que cualquier espacio del centro puede ser un magnifico recurso para el desarrollo y el aprendizaje de los alumnos. (Trujillo, 2014, p. 20)

Por lo anterior se puede afirmar que debe haber un equilibrio entre el espacio simbólico y el físico. Por un lado, debe contar con un lugar físico donde la escuela pueda desarrollarse y por el otro, debe aprovechar la riqueza biocultural del resguardo como ambiente escolar, nutriendo el simbólico con la memoria oral.

Al observar las condiciones de la caseta comunitaria y comprender lo que expone la profesora, se puede afirmar que las condiciones del lugar donde se dictan las clases afectan de forma considerable la calidad de la formación que pueden recibir los niños y niñas Emberas de la comunidad de Chigorodocito. Sin embargo, al entender el ambiente escolar como un espacio simbólico capaz de desarrollarse en diversos escenarios de la comunidad o resguardo, se puede recomendar que las y los maestros Embera de esta comunidad, dispongan de los diversos contextos naturales existentes en la comunidad: ríos, sitios sagrados, selvas y escenarios adecuados para realizar una praxis pedagógica cercana a lo expuesto por el Sistema de Educación Indígena Propio (SEIP).

Otro elemento importante de la entrevista giró en torno a la distancia entre los valores culturales y los impartidos por la es- cuela. La entrevistada afirmó que los valores culturales son aprendidos en la casa y los elementos de lectura y escritura se aprenden en las escuelas. Lo anterior se contrapone a los sustentos pedagógicos del Sistema Indígena de Educación Propia donde se manifiesta la necesidad de que las escuelas indígenas lideren procesos formativos articulados a los conocimientos ancestrales, la autonomía y aspectos políticos.

Por su parte, la maestra expresa que las prácticas comunitarias culturales están siendo transformadas y olvidadas, lo que permite preguntar lo siguiente: Si las prácticas culturales comunitarias son elementos pedagógicos significativos para que los niños Embera puedan aprender sobre la sabiduría ancestral ¿cómo y cuándo los niños y niñas Embera adquirirán el legado ancestral de su cultura si estos escenarios son relegados y olvidados? Por este motivo, el movimiento indígena de Antioquia viene pensando en la implementación del SEIP para que la escuela ayude a fortalecer los conocimientos ancestrales y sea un puente para difundirlos ¿estaría la escuela preparada para asumir esta labor?, ¿cómo entienden los maestros indígenas la articulación de la escuela y la comunidad?

En definitiva, para que el movimiento indígena de Antioquia pueda cumplir a cabalidad el sueño de ver al SEIP desarrollándose en las escuelas indígenas de Chigorodó, es necesario fortalecer la formación de los docentes, propiciar espacios críticos, comunitarios, políticos y de ritualidad espiritual, que ayuden a dinamizar su praxis pedagógica y didáctica.

La implementación de la Educación Propia en las escuelas indígenas debe partir de una relación armónica y colaborativa entre las sabias y sabios con la Organización 
Indígena de Antioquia (OIA), la Secretaria de Educación Departamental, los Cabildos Mayores Indígenas de los municipios y los docentes. También, es necesario garantizar espacios críticos donde los maestros articulen su sabiduría ancestral con su quehacer docente.

En este sentido, es importante reconocer las orientaciones del programa de la Licenciatura en Pedagogía de la Madre Tierra de la Universidad de Antioquia, escuchadas en transcurso de nuestra formación:

Volver a escuchar, observar, tejer, reconocer la sabiduría de las abuelas y abuelos de nuestras comunidades, vincular sus conocimientos a las prácticas formativas de las escuelas indígenas y así, analizar colectivamente el daño que la cultura occidental viene ejerciendo contra las prácticas ancestrales, buscando herramientas para sanar la herida colonial que hoy se reviste de modernismo.

En esta búsqueda de sanación la escuela debe cumplir una función transformadora. En palabras de Paulo Freire (1969), debe hacer práctico su papel comunitario.

Otro aspecto para rescatar de la entrevista es la manifestación de la ausencia de material didáctico de la Educación Propia. La escuela no cuenta con suficientes insumos pedagógicos que rescaten y visibilicen el pensamiento Embera. Al respecto, los maestros quieren que existan elementos concretos para aplicar la Educación Propia en su praxis, tener libros y guías. Empero, si está pidiendo que le creen sus herramientas prácticas: ¿dónde queda el rol creativo del maestro indígena?, ¿es posible que la educación conductista sesgara la práctica de los docentes indígenas de la escuela de Chigorodocito?, ¿por qué nos conformamos con seguir el patrón y replicar lineamientos hechos por otros?

Un último elemento que se puede inferir de este diálogo se relaciona con la posición manifestada por la docente cuando afirma que los cantos ancestrales son recursos que pueden ayudar a enseñar sobre la cultura Embera y sobre las historias de la vida del pensamiento indígena. Para ella, esta es una práctica cultural que puede articularse a la escuela como elemento artístico que fortalezca la identidad y la apropiación cultural.

En este ámbito, la Educación Propia en las comunidades indígenas de Chigorodó se encuentra descontextualizada del saber de los maestros, sin recursos didácticos y pedagógicos para su ejecución y en tensión con las prácticas culturales del pueblo, pero con intereses de aprender, comprender y construir los tejidos para que pueda urdirse una educación sensible, critica, artística para las y los niños del pueblo Embera Eyábida. Es urgente prestar atención a lo que proponen los maestros de las comunidades indígenas, porque al final ellos son los actores más cercanos a los niños y niñas; se debe atender sus necesidades, falencias y exigencias y sobre todo, acompañar su praxis pedagógica.

Es evidente que los conocimientos ancestrales están siendo sustituidos por elementos de la cultura occidental y antes de exigirle a la escuela que ayude a solucionar este problema se debe preparar, sanarla de la herida colonial y tomar el tiempo para zurcir e hilar la Educación Propia en el territorio Embera Eyábida. Este es un reto que deberá apoyarse en la sabiduría de las y los abuelos para que la cultura Embera se conserve y proteja y así, las niñas y niños disfruten de elementos pedagógicos y culturales que visibilicen la sabiduría de sus mayores. 
Por lo tanto, se hizo un llamado propositivo a la escuela y a su acción pedagógica dentro de los territorios Embera de Chigorodó para que revindicaran y abrieran caminos que vinculasen la sabiduría de los cantos ancestrales en su currículo.

En este sentido, se realizaron talleres formativos sobre los cantos ancestrales con niños y niñas de primero y segundo de primaria, se crearon cuentos relacionados con la vida de las abuelas conocedoras de los cantos y se presentó una película de animación titulada "La historia de origen de la creación del agua del pueblo Embera Eyábida".

\section{Chimbizu Atei Juruida: El vuelo del Colibrí como método de investigación Embera}

Para el pueblo Embera, el Colibrí es un ave llena de una inmensa sabiduría. En la historia de origen del agua (Jaramillo, 2011) , Kagarabí, el dios Embera, delegó al colibrí para que rastreara e indagara a Jenzera ${ }^{7}$. Esta ave estuvo buscando y rastreando hasta que encontró el árbol de Jenené, donde Jenzera tenía escondido el agua. El colibrí siguió a esta hormiga de fuego y descubrió dónde guardaba el agua que negaba al pueblo Embera.

Se retoma el vuelo y rastreo del Colibrí como una metáfora que acerca desde la historia de origen a la Juruida Embera. Estar en constante atención y vuelo, escuchar las palabras de los sabios, observar las huellas del camino y buscar con calma, son los métodos investigativos que propone esta ave a través de las características que alberga en la historia de origen del agua. Es decir, Juruida se relaciona con el alma de colibrí, ave viajera, aventurera, dulce y sabia.

\section{Al respecto el CMICH (2013) señala} que:

Para nosotros los Embera, es muy importante conocer y valorar el legado cultural que dejaron nuestros ancestros, legado que se ha ido transmitiendo de generación, en generación. Para acercarnos a este conocimiento debemos saber rastrear toda la sabiduría y conocimientos que hay en nuestras comunidades y aprender del Chimbuzú quien fue enviado por el dios Karagabi a rastrear donde escondía el agua Jenzerá - La hormiga. El colibrí, en esta importante tarea de rastrear, se dispuso a observar con calma, a escuchar con atención, a conocer muy bien el contexto, a poner en práctica todo lo que Karagabi le había enseñado y a mirar las huellas en el camino. (p.20)

Inicié esta investigación para acercarme a mi pueblo Embera Eyábida, volver como el Colibrí que, aunque vive de viaje $\mathrm{y}$ en constantes vuelos buscando néctares, siempre retorna a su nido a brindarle a su hembra, a su macho o a sus pichoncitos la dulzura encontrada en las flores. Los cinco años dentro de la academia y los viajes a otros pueblos me habían transformado; podía sentir un profundo arraigo al vivir en comunidad, pero también una visión crítica me acompañaba.

Durante los primeros días en la comunidad descubrí que poseía modos distintos de mirarme en territorio. Era como si mi ombligo me llamara a reconocerme y sentirme en los conocimientos de las mujeres Embera, pero había algo que me dolía, que me hacía ruido. Era el resultado de algunas injusticias que, desde mi óptica, no podían categorizarse como ancestrales, ni cosmogónicas. Para mí no era natural el abandono que los abuelitos estaban viviendo, ni la violencia contra la mujer, ni la deforestación 
de los bosques que los mismos hombres de la comunidad hacían. No podía dar por hecho una situación que era reciente, resultado de décadas de aculturación, colonización y neoliberalismo.

Nuestros abuelos y nuestras abuelas conocían el territorio tranquilamente, lo conocían por medio de las enseñanzas de sus madres, padres y familiares y de su profunda observación e interacción con la naturaleza. En el territorio, nuestros abuelos y abuelas realizaban las actividades diarias de su cultura en un entorno de tranquilidad y sabiduría, sin tantos conflictos con agentes externos como los kabaramia o kapuria (colonos, no indígenas). (Domicó, 2010, p.35)

Domicó (2010) realizó un trabajo valioso en su tesis de maestría. Detalló algunas prácticas culturales del pueblo Embera Eyábida, evidenciando que los abuelos poseían grandes enseñanzas y eran respetados. De igual forma, se valoraba el médico tradicional o Jaibaná, autoridad espiritual de la comunidad que en la actualidad ha perdido legitimidad por las imposiciones de las iglesias, al principio de la católica y en la actualidad, del cristianismo evangélico.

Al estar en comunidad y ver que muchas de sus flores estaban perdiendo dulzura y belleza, me llené de conflictos y pude sentir e identificar todo el daño de la colonización, sentir la herida dejada por el despojo y la violencia que no estaba sanada desde la raíz. Sus consecuencias se podían ver en el desinterés de las juventudes por practicar la sabiduría de los ancestros, en el olvido de los adultos y en el descuido a las nuevas generaciones. Tropecé con la herida que hacía parte de mí y comenzó a sangrar; allí reconocí el valor de las sanadoras y sanadores que han sido esenciales en la historia de nuestro pueblo Embera: las abuelas y abue- los. ¿Si dejamos que sus voces se apaguen qué pasará con la herida?

Estando en comunidad comprendí que la investigación debe ser y vivirse con el pueblo, que requiere más de nuestra atención y fuerza para que siga surgiendo y zurciéndose.

Muchos elementos reflexivos se nos presentaron en este camino de exploración de las nociones de la Juruida Embera. Desde mi vuelo se resalta que:

- Las Juruida Embera deben partir de las necesidades del contexto. En este esquema no es tan relevante porqué se investiga sino el para qué. ¿Qué aportes se pueden compartir con la comunidad? En este proceso el fin último no es la investigación sino la semilla o fruto que se deja sembrado, es decir, más que un fin, la investigación es un medio que ayuda a sembrar y recoger cosechas más saludables para la comunidad. Al respecto, Smith (2016) afirma que "En muchos proyectos el proceso es mucho más importante que el resultado. Se espera que los procesos sean respetuosos, que brinden capacidades a la gente para sanar y educar" (p, 175).

- La Juruida en comunidad es flexible, como los ríos cristalinos que adornan nuestras tierras, no tienen linealidad. Sus cuerpos son siluetas en movimiento que se compactan con los elementos que lo componen, así es la investigación en nuestros pueblos, va creando su cuerpo al accionarla en territorio.

- Sin duda alguna la Juruida Embera es colectiva y colaborativa, resultado de la confianza que fue gestándose al conocernos como equipo. Se puede afirmar que la Juruida Embera tiene cercanía a los postulados de la investigación colaborativa que exaltan Boavida y da Ponte (2002): 
Un tema recurrente en la literatura sobre colaboración es la necesidad de la confianza (véase, por ejemplo, Hargreaves, 1998). Para muchos autores, la confianza es "el primer paso para la co- laboración" (Goulet y Aubichon, 1997, p 118), desarrollándose esta confianza en un clima de respeto y atención, que se manifiesta tanto en los ámbitos personal como profesional. $(\mathrm{p}, 130)$

\section{Conclusión}

Para el pueblo Embera el canto ancestral es una práctica cultural significativa porque permite la conservación y transmisión de los conocimientos ancestrales; es un acto pedagógico que las sabias y los sabios han utilizado desde antaño para enseñar el pensamiento originario a las generaciones jóvenes. Esta práctica se encuentra en peligro de desaparecer con la muerte de las abuelas. Por lo tanto, las apuestas de la Educación Propia deben retomar y fortalecer la sabiduría de los truambis para aportar a su permanencia y continuidad.

La mujer Embera ha sido milenariamente una guerrera y en la actualidad se encuentra inmersa en diversos escenarios políticos exigiendo las reivindicaciones de sus derechos y el respeto a la Madre Tierra. Las acciones depredadoras del hombre actual ponen de relieve una pérdida de respeto al vientre de la tierra y una acción de inseminación a la matriz que mantiene la vida. Cada día vemos cómo los hombres se pierden en guerras, muertes y destrucciones, sumidos en lógicas patriarcales que silencian el canto armonioso de la mujer y de la naturaleza. Es necesario comenzar diálogos de saberes, resignificar y avivar las acciones transformativas que las mujeres Embera Eyábida realizan en sus territorios para permitir la continuidad de la esencia del pueblo.

La mujer Embera Eyábida, al igual que la tierra, se encuentra manifestando su des- acuerdo con el mundo patriarcal que por décadas la ha silenciado, ha explotado y maltratado su cuerpo que representan los recursos naturales y orgánicos que nos habita. Tal y como decía el profesor Abadio Green:

Todo lo que le está ocurriendo a la tierra lo está sintiendo la mujer; la insensatez del hombre al maltratar y lastimar a la tierra le está haciendo daño físico también a las mujeres. Mira que cada día están aumentando los cánceres de útero, no nos estamos dando cuenta que la tierra que representa la mujer está sintiendo las consecuencias de violencia, extracción y daño a la tierra, tenemos que cambiar eso, mirar a la tierra con el amor como nos miran nuestras madres o abuelas, debemos comenzar a sanar todo el daño que hemos depositado en ella. (Comunicación personal. Octubre 8 de 2017)

Las mujeres indígenas estamos despertando y reviviendo las voces de las luchas y enseñanzas de nuestras guerreras antiguas, ellas son el espíritu que alientan nuestros pensamientos y gracias a ellas podemos enunciar nuestras voces. Gracias entonces a Anacaona, Gregoria Apaza, Bartolina Sisa, Kurusa Llave, Micaela Bastidas, Huillac Ñusca, Angata, Janequeo Guacolda, Fresia, Tegualda, Lorenza Abimañay, Rosa Señapanta, Margarita Ochoa, Baltazara Chiza, Margarita Pantoja, Gaitana, Ague, Ayunga y otras mujeres más recientes como Berta Cáceres, Ana Ochoa, Teresita Bailarin, Anita 
Bailarin, que han ofrendado sus vidas para que nosotras pudiésemos existir más sabias, libres y guerreras. A ellas, nuestras ances- tras, estas palabras que salen desde mi útero porque me han inspirado a parir este trabajo.

\section{Notas}

1. Jaibanà: Médico Tradicional

2. Jaibanawera: Mujer Médica Tradicional

3. Debido a un acceso más cercano a la cultura occidental, los hombres están dejando de lado elementos esenciales de la cultura Embera, entre ellos, el truambi y las danzas tradicionales.

4. Principios Pedagógicos de la Licenciatura en Pedagogía de la Madre Tierra. Silencio: El silencio como posibilidad de escucharnos y de escuchar. Lo que conocemos como "occidente" hace demasiado ruido, la academia está llena de palabras, de verdades. El silencio como categoría pedagógica invita a mirar hacia dentro, invita a conocer de otro modo y reconocer que a través del silencio consciente han pervivido conocimientos y pueblos ancestrales. El silencio es una construcción permanente y hace parte de la forma de conocer en la licenciatura en Pedagogía de la Madre Tierra.

5. El Jai traduce al español "espíritu”. Para el embera, la gente puede enfermar de Jai y consiste en que el cuerpo es controlado por energías negativas que pueden hacerle daño y forzarlo a actuar de manera incomprensible para los demás.

6. Comité de Mujeres Indígenas: Las mujeres indígenas de Chigorodó comienzan a reunirse alrededor de proyectos productivos (crianza de gallinas, siembra de alimentos, etc.). En esos espacios comenzaron a hablar sobre las problemáticas de violencia intrafamiliar. "Empezamos a decir que nuestras opiniones eran importantes y así empiezan las mujeres. Acá en Chigorodó hay que reconocer que el liderazgo masculino de alguna manera también ha tenido esa voluntad política de vincular a las mujeres en el quehacer político. El comité de mujeres ha tenido algunos avances institucionalizando una asamblea anual donde se discuten diferentes temáticas; nos reunimos alrededor de las problemáticas de las mujeres, pero también entorno a lo que pasa con el territorio, la salud" (Casama, T. Comunicación personal, 2018). Actualmente, el comité cuenta con un consejo municipal donde hay representantes por cada comunidad y una presidenta llamada Sirley Domicó. Este comité se encarga de realizar y monitorear las denuncias de violencias ejercidas en contra de las mujeres indígenas.

7. Jenzera: La hormiga que escondió el agua del pueblo Embera en la historia de origen.

\section{REFERENCIAS}

Bailarín, M. (septiembre 9 de 2017) Entrevista en el marco del trabajo de campo de la comunidad indígena de Chigorodocito. Archivo personal 
Bermúdez, E. (1987). Música Indígena Colombiana. Maguare, Revista del Departamento de Antropología de la Universidad Nacional de Colombia (No 5)

Boavida, A. y Ponte, J. (2011) Investigación colaborativa: Potencialidades y problemas. Revista Educación y Pedagogía, 23(59), 125-135.

Casama, T. (febrero 15 de 2018) Entrevista en el marco de la VIII Asamblea de mujeres indígenas de Chigorodó. Archivo personal.

Cabildo Mayor Indígena de Chigorodó -CMICH-. (2013). Lo que los Ancestros nos dejaron: Nuestra identidad Embera en Chigorodó. Chigorodó, Colombia: Organización Indígena de Antioquia

Domicó, G. (2010). Urikabaita jarakampe sabua chupanuta yumachirata yuberaume dalli embera nemburuadeba en la Comunidad Indígena Chuscal Tugurido de Murrí, municipio de Frontino Colombia (Tesis de Maestría). Universidad de Antioquia. Medellín

Domicó, G. (septiembre 8 de 2017). Entrevista en el marco del trabajo de campo de la comunidad indígena de Chigorodocito. Archivo personal

Domicó, J (septiembre 20 de 2017). Entrevista en el marco del trabajo de campo de la comunidad indígena de Chigorodocito. Archivo personal

Green, A. (2011). Significados de vida: Espejo de nuestra memoria en defensa de la Madre Tierra (Tesis Doctoral). Universidad de Antioquia. Medellín

Green, A. (octubre 8 de 2017). Comunicación personal en el marco de reunión con el grupo de investigación Diverser. Universidad de Antioquia.

Jaramillo, E (2011). Kimy, palabra y espíritu de río. Bogotá, Colombia. Editorial Códice Ltda.

Quijano, A. (Lander, E) (2000). La colonialidad del saber: eurocentrismo y ciencias sociales. Perspectivas Latinoamericanas. Buenos Aires, Argentina: CLACSO, Consejo Latinoamericano de Ciencias Sociales

Sacavino, S. (2017). Tecidos feministas de Abya Yala: Feminismo Comunitário, Perspectiva Decolonial e Educação Intercultural. Uni-pluriversidad, 16(2), 97-109.

Smith, L. (2016). A descolonizar las metodologías. Investigación y Pueblos Indígenas. Santiago, Chile: LOM Ediciones

Trujillo, L. (2014). La importancia de los espacios escolares en la enseñanza/aprendizaje de los alumnos (Tesis de Maestría). Universidad de Málaga. Málaga, España 